Objectives Our aim was (a) to assess the content validity of a Thai translation/version of a quality of work-life evaluation tool, and (b) to examine its accuracy vis-à-vis nursing in Thailand.

Method Descriptive correlation study

Forward-backward translating procedures were used to develop the Thai version of the work-related quality of life scale. Six nursing experts participated in assessing content validity and 374 registered nurses (RNs) participated in its testing. After a two-week interval, 67 of the RNs were retested. Structural validity was examined using principal components analysis and the Cronbach's alphas calculated. The respective independent sample $t$-test and intra-class correlation coefficient were used to analyse known-group validity and test-retest reliability.

Sample group: Cluster sampling was used to select 374 registered nurses from the In- and Out-patient Departments t Srinagarind Hospital, Khon Kaen University.

Results The content validity index of the scale was 0.97. Principal components analysis resulted in a seven-factor model, explaining 59\% of total variance (Cronbach's alpha for the subscales ranged between 0.65 and 0.84 , while the overall Cronbach's alpha was 0.925). The known-group validity was established in the assessment results of the difference in bureaucrats (civil servants) vs. casual employees by $\mathrm{F}(8.855,0.003)$ and t $(3.305, \mathrm{p}<0.01)$. Apparently, government employees have a better quality of work life than the university's casual employees. Good test-retest reliability was observed $(r=0.898, p<0.01)$. Conclusions The Thai version of a work-related quality of life scale appeared to be well validated and therefore useable for determining the quality of work-life among nurses in Thailand.

\section{A COHORT STUDY OF WORKERS EXPOSED TO PFOA}

Kyle Steenland, Liping Zhao, Andrea Winquist. Rollins School of Public Health, Emory U, Atlanta, Ga, USA

\subsection{6/oemed-2014-102362.170}

Objectives PFOA has been linked to several diseases, but findings are inconclusive, and there have been no incidence studies in worker populations.

Method We interviewed 3700 workers or their next-of-kin in 2009-2011 for medical history, and sought medical records to validate self-reported disease. A job-exposure matrix based on over 2000 PFOA serum measurements was used to estimate serum levels over time for each worker, while a separate estimation was made for non-occupational exposure due to drinking PFOA-contaminated water. Cumulative dose in the serum (ng/ml-years) was the metric of interest. We studied 18 disease outcomes with 15 to 1430 cases; analyses were limited to confirmed cases.

Results The median year of birth was 1951; 5\% had died. The median measured serum level was $113 \mathrm{ng} / \mathrm{ml}$ in 2005 ( $\mathrm{n}=$ 1900), compared to $4 \mathrm{ng} / \mathrm{ml}$ in the US population. Among 18 outcomes studied, only ulcerative colitis (10 year lag) showed a significant trend with increasing exposure $(\mathrm{p}=0.05)$ (RRs by quartile $1.00,3.00,3.26,6.57, \mathrm{p}$ value trend 0.05$)$, similar to earlier findings in a community cohort study in the same area. Positive but non-significant trends were also observed for prostate cancer and non-hepatitis liver disease, and female hypothyroidism, which have been implicated in other studies, No marked trends were seen for high cholesterol, which had been seen in the community study.

Conclusions Ulcerative colitis was linked to PFOA exposure among workers. Analyses of other diseases did not show marked trends. Data were limited by small numbers, a largely survivor cohort, and few low exposed referents.

\section{EXPOSURE-RESPONSE ANALYSES FOR SOLUBLE PLATINUM-SALT EXPOSED WORKERS AND SENSITISATION: A RETROSPECTIVE COHORT STUDY AMONG NEWLY EXPOSED WORKERS USING ROUTINELY COLLECTED SURVEILLANCE DATA}

${ }^{1}$ Dick Heederik, ${ }^{1}$ Jose Jacobs, 'Sadegh Samadi, 'Lutzen Portengen, ${ }^{2}$ Frits van Rooy, ${ }^{3}$ Remko Houba. ${ }^{1}$ Institute for Risk Assessment Sciences, Utrecht, The Netherlands; ${ }^{2}$ Arbo Unie, Expert Center for Chemical Risk Management, Utrecht, The Netherlands; ${ }^{3}$ Netherlands Expert Center Occupational Respiratory Diseases, Utrecht, The Netherlands

\subsection{6/oemed-2014-102362.171}

Objectives Soluble platinum salts are well known respiratory sensitising agents leading to work related sensitisation in the work environment. No quantitative exposure response relation has been described for soluble platinum salts. The objective of this study was to explore exposure response relations for soluble platinum salt exposed workers.

Method A retrospective cohort study was conducted using routinely collected health surveillance data and soluble platinum exposure data. Workers who newly entered between 1 January 2000 and 31 December 2010 were included and the relation between measured soluble platinum exposure and sensitisation (as determined by skin prick testing) was analysed in more than 1000 refinery workers from 5 refineries from whom a total of more than 1700 personal exposure measurements were available. Exposure response relations were analysed in survival analysis considering changes in exposure over time. Associations were explored for present exposure, cumulative exposure and average exposure. The exposure was lagged by $0.5,1.0,1.5$, etc. year with a maximum of 5 years.

Results A clear exposure response relation was observed, most strongly for present platinum salt exposure. Exposure lagging showed that exposure preceding sensitisation with maximally $1-$ 2 years was most strongly associated with sensitisation risk. The exposure response relationship was modified by smoking and atopy, but relative risks for smoking and atopy were only modestly elevated.

Conclusions The precision of estimate of the exposure-response relationship derived from this dataset appears superior to previous epidemiologic studies conducted on platinum salt sensitisation and may as a result have possible utility to occupational exposure standard setting.

\section{CHRONIC PROBABLE POSTTRAUMATIC STRESS DISORDER AMONG POLICE REGISTRANTS IN THE WORLD TRADE CENTRE HEALTH REGISTRY TEN YEARS AFTER 9/11/01}

${ }^{1}$ James Cone, ${ }^{2}$ Rosemarie Bowler, ${ }^{1}$ Jiehui Li, ${ }^{3}$ Erica Kornblith, ${ }^{4}$ Annum Shaikh, ${ }^{2}$ Vihra Gocheva. 'New York City Department of Health and Mental Hygiene, New York, NY, USA; ${ }^{2}$ San Francisco State University, San Francisco, CA, USA; ${ }^{3}$ California School of Professional Psychology, Alliant University, San Francisco, CA, USA; ${ }^{4}$ Emory University School of Public Health, Atlanta, GA, USA

10.1136/oemed-2014-102362.172

Objectives The World Trade Centre Health Registry (WTCHR) prospectively follows a cohort of over 71000 individuals who reported being directly exposed to the $9 / 11 / 01$ terrorist attack. 